\title{
Malignant granular cell tumor with breast metastasis: A case report and review of the literature
}

\author{
JIDA CHEN $^{1}$, LINBO WANG $^{1}$, JING XU $^{2}$, TAO PAN $^{1}$, JIANGUO SHEN $^{1}$, WENXIAN HU $^{1}$ and XIAOMING YUAN ${ }^{1}$ \\ Department of ${ }^{1}$ Surgical Oncology, and ${ }^{2}$ Pathology, Sir Run Run Shaw Hospital, College of Medicine, \\ Zhejiang University, Hangzhou, Zhejiang 310016, P.R. China
}

Received February 16, 2012; Accepted April 24, 2012

DOI: $10.3892 / \mathrm{ol} .2012 .702$

\begin{abstract}
Malignant granular cell tumors (MGCT) are rare mesenchymal soft tissue neoplasms of Schwann cell origin without adequate follow-up. This study describes a case of MGCT with right breast metastasis following two local recurrences. The patient consented to a right breast lumpectomy with right axillary dissection, a right abdominal wall lumpectomy and a right inguinal lumpectomy with dissection. Pathological examination revealed that the two initial lesions were consistent with benign histological performance. The later lesions were classified as malignant due to the observation of spindling of the tumor cells, vesicular nuclei with large nucleoli and increased mitotic rate. Immunohistochemical study of the lesions revealed positivity for $\mathrm{S} 100$ protein. The Ki-67 proliferation index increased from 1 to $10 \%$. Twenty-seven months after surgery, the patient was in good health with no sign of further tumor development. We recommend wide local excision with regional lymph node dissection as the first choice of treatment for MGCT.
\end{abstract}

\section{Introduction}

Granular cell tumors (GCT), first described by Abrikossoff in 1926, are an uncommon mesenchymal soft tissue neoplasm of Schwann cell origin $(1,2)$. These tumors may occur throughout the body, usually in the head and neck, skin or subcutaneous tissues of the trunk and upper extremities, breasts and female genital region. They are usually benign and solitary; however, approximately $2 \%$ occur as malignant tumors, and $5-10 \%$ as multiple lesions $(3,4)$. The common sites for distant metastases include bone, peripheral nerves, the peritoneal cavity and the lung (5). In 1992, Uzoaru et al reported a unique case of MGCT with breast cancer without adequate follow-up (6). For MGCT, surgical excision is the only treatment method proven

Correspondence to: Professor Linbo Wang, Department of Surgical Oncology, Sir Run Run Shaw Hospital, College of Medicine, Zhejiang University, Hangzhou, Zhejiang 310016, P.R. China

E-mail:wanglinbo@medmail.com.cn

Key words: malignant granular cell tumor, metastasis, breast to be effective. In the present study, we report a case of MGCT with breast metastasis and discuss the available treatment modalities. The study was approved by the ethics committee of Sir Run Run Shaw Hospital, Zhejiang University, China. Consent was obtained from the patient.

\section{Case report}

In October 2009, a 56-year-old Chinese woman was referred to the Department of Surgical Oncology at Sir Run Run Shaw Hospital due to multiple painless masses in the right lower abdominal wall, right groin and right breast. A mass in the right lower abdominal wall had first appeared 10 years earlier, and had been excised in 2003. The tumor recurred in 2004 and was again excised in 2006. The patient developed a mass in the same region of the abdominal wall once again in 2007, followed by a mass in the right groin and right breast in 2008 . On physical examination, masses were present in the upper outer quadrant of the right breast, right lower abdominal wall and right groin, measuring $3 \times 3,5 \times 8$ and $6 \times 7 \mathrm{~cm}$ in size, respectively (Fig. 1A). These masses were fixed, non-tender and firm on palpation. The abdominal wall mass, whose overlying skin was red and crusted, was located in the interior of the incision scar. There was a swollen lymph node $2 \mathrm{~cm}$ in diameter in the right axilla, which was freely mobile and slightly hard. Ultrasound and X-ray revealed that the breast mass was a carcinoma. Supraclavicular lymph nodes, pelvic lymph nodes, liver, lungs and bones were evaluated preoperatively to exclude metastasis. Levels of tumor markers, including CA153, CA125 and CEA, were normal. A puncture biopsy of the right breast mass was performed and confirmed the mass as a GCT. A right breast lumpectomy with right axillary dissection, a right abdominal wall mass resection, and a right inguinal mass resection with inguinal dissection were performed. Twenty-seven months after surgery, the patient was in good health with no sign of further tumor development.

Gross examination revealed that the mass in the right breast, right lower abdominal wall and right groin measured 2.8x2.5, 6x3.3 and 5.2x4 cm in size, respectively (Fig. 1B-D). The masses were yellow-gray in color and well-circumscribed. The previous two biopsy specimens resected in 2003 and 2006 were reviewed. Having similar histological performance, the specimens demonstrated a pattern compatible with GCT. The lesion consisted of nests of polygonal cells with generally 
A

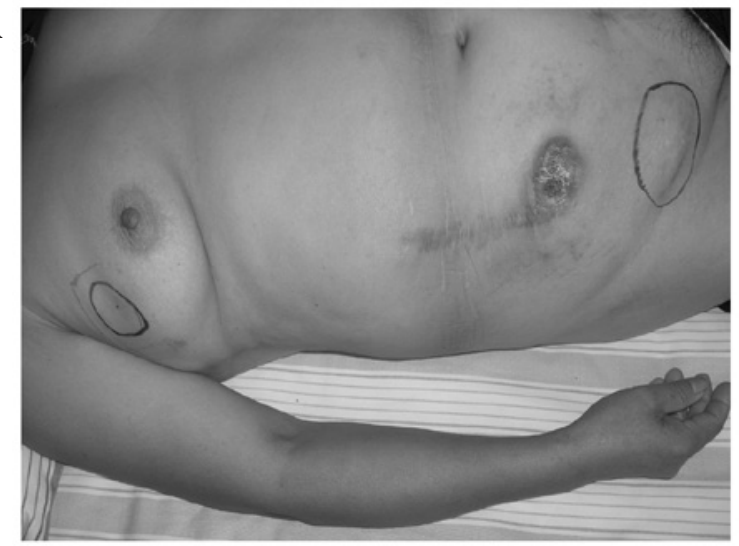

$\mathbf{C}$

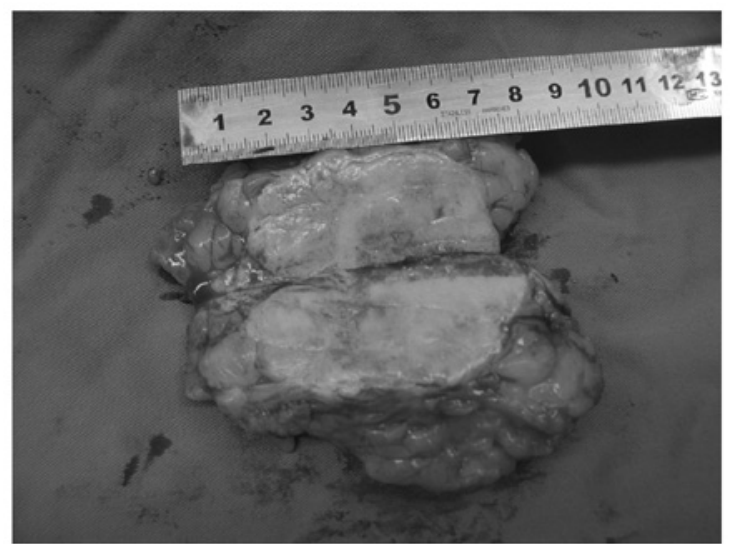

B

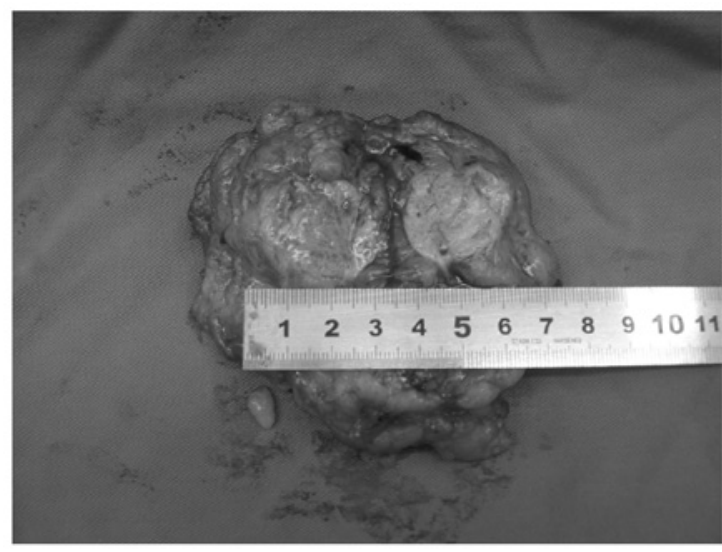

D

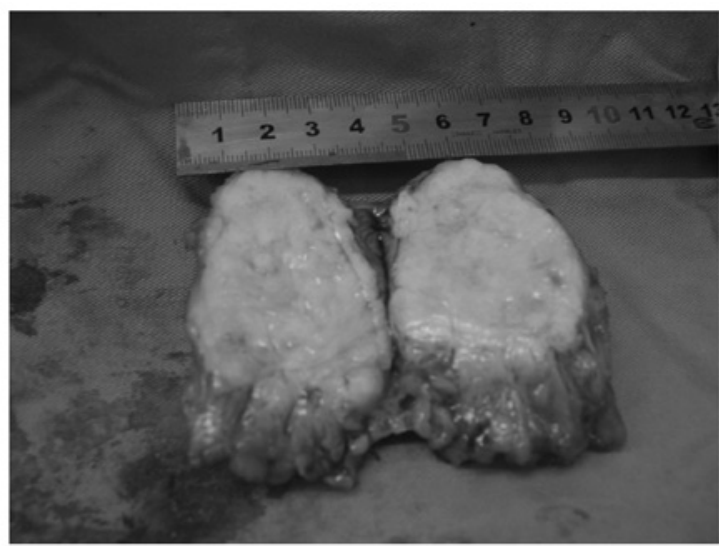

Figure 1. (A) Clinical image. (B-D) The cut surface of the resected specimens: (B) right breast; (C) abdominal wall; (D) right groin.

A

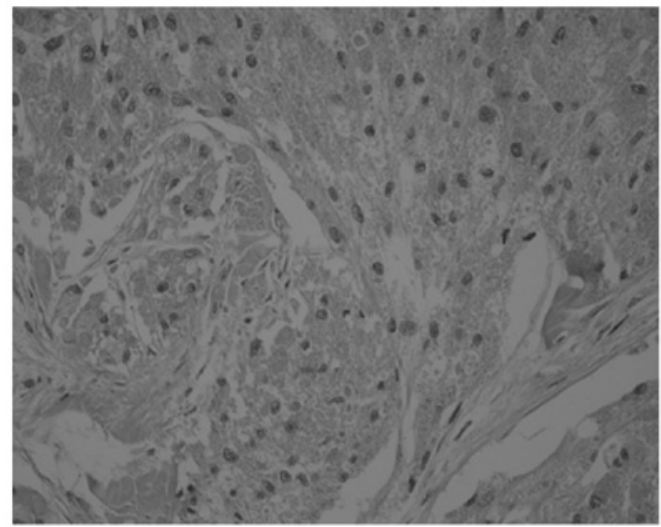

C

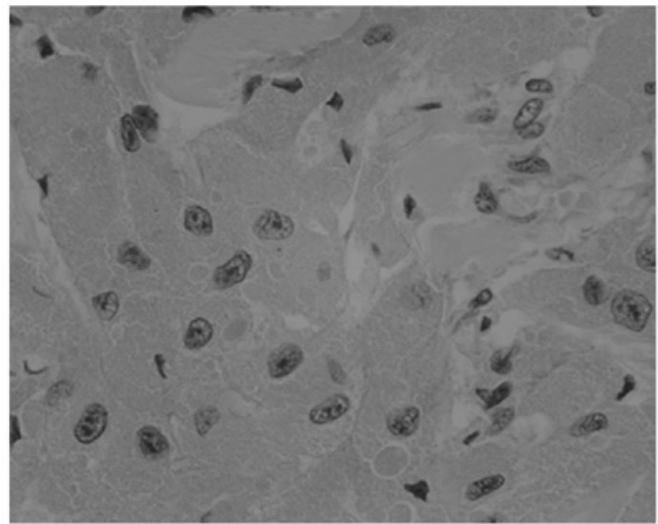

B

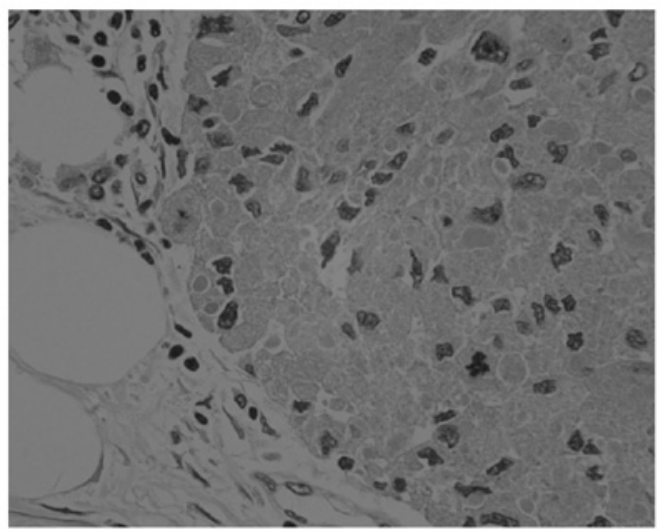

D

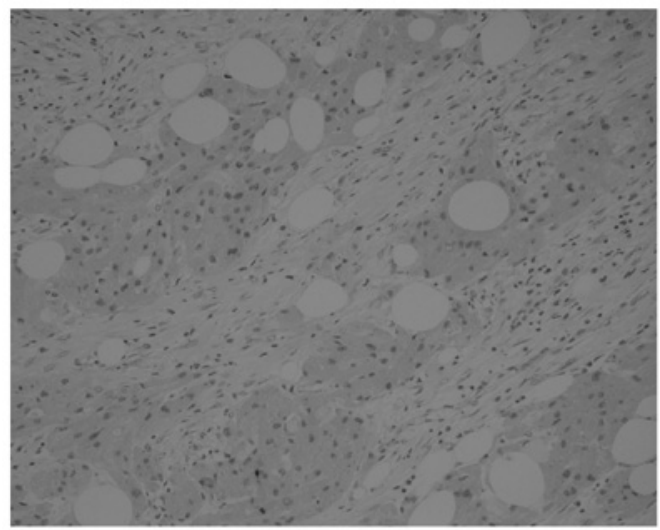

Figure 2. (A-D) Histological appearance of the granular cell tumor. (A) The 2006 abdominal wall mass (H\&E; original magnification, x200). (B) The right breast mass (H\&E; original magnification, x400). (C) The 2009 abdominal wall mass (H\&E; original magnification, x400). (D) Right groin mass (H\&E; original magnification, $\mathrm{x} 100)$. 
A

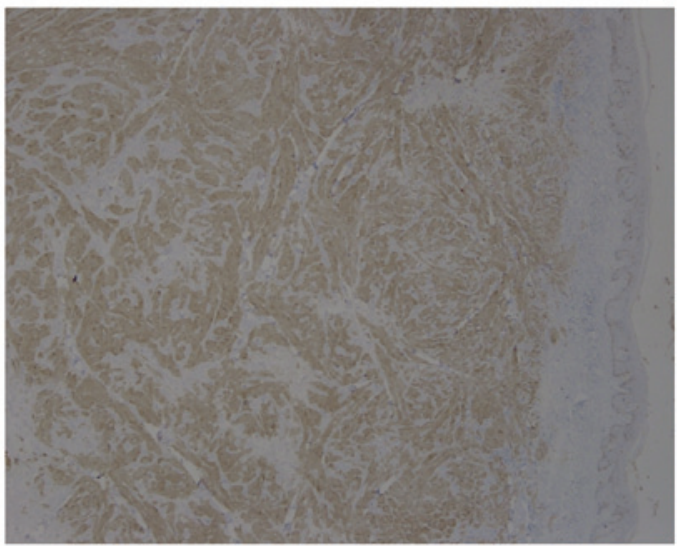

B

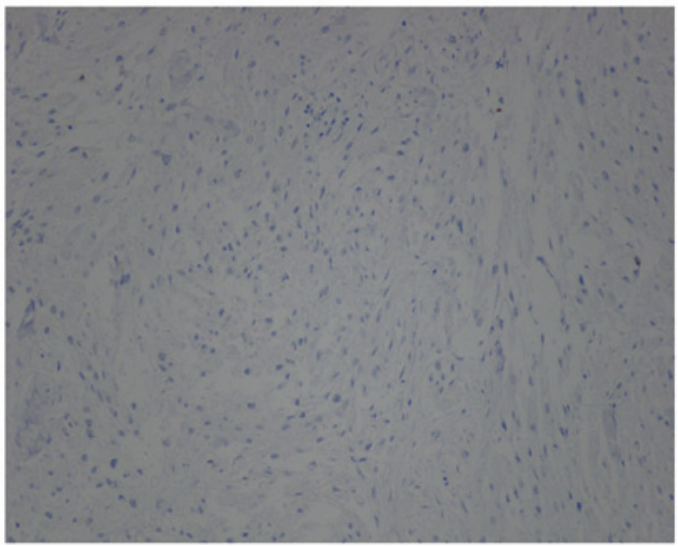

C

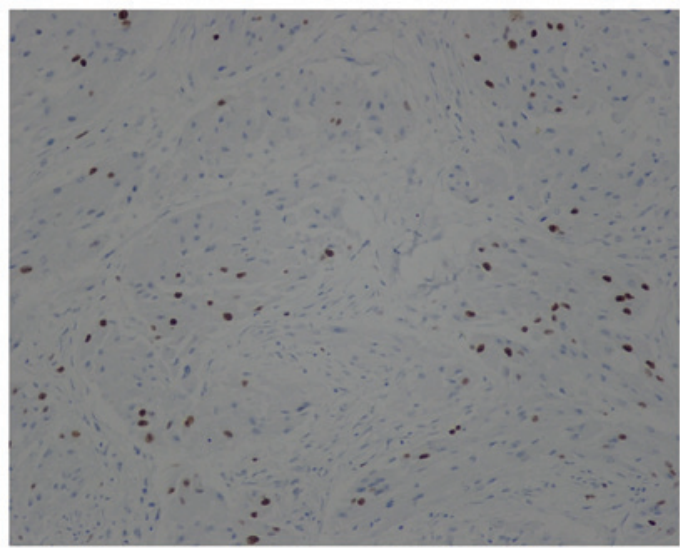

Figure 3. (A) Nuclear and cytoplasmic staining with antibodies to S100 protein in the 2009 abdominal mass (original magnification, $\mathrm{x} 200$ ). (B) Immunohistochemical staining for Ki-67 of the 2006 abdominal wall mass (original magnification, $\mathrm{x} 100$ ) and (C) the 2009 abdominal wall mass (original magnification, $\mathrm{x} 100$ ).

round nuclei and abundant eosinophilic granular cytoplasm. The mitotic count was 1 per 10 high-power fields (magnification, x200). No cytologic pleomorphism, vesicular nuclei with prominent nucleoli or areas of necrosis were observed, and no increase in nuclear to cytoplasmic ratio $(\mathrm{N}: \mathrm{C})$ was noted. The tumor nests were surrounded by abundant reactive fibrous stroma, plasma cells and scattered groups of lymphocytes (Fig. 2A). The findings concurred with the diagnosis of granular cell tumor. The pathological appearance of the 2009 abdominal wall, right breast and right inguinal region masses were similar (Fig. 2B-D). The tumors were arrayed in nests and sheets composed of round to irregular shaped cells with abundant eosinophilic granular cytoplasm. The lesions were not completely encapsulated and focally invaded adjacent connective tissue. The tumors demonstrated mild cytologic pleomorphism, and round to oval-shaped vesicular nuclei with prominent nucleoli were observed. The mitotic count was 3 per 10 high-power fields (magnification x200). Four of the 14 right axillary lymph nodes were found to be metastatic. The lymph nodes in the right inguinal region demonstrated reactive hyperplasia.

Immunohistochemical study of all tumors revealed positivity for S100 protein, and p53 in $<5 \%$ of cells. The Ki- 67 proliferation index was $1 \%$ in the 2003 and 2006 biopsies and $10 \%$ in the 2009 biopsy. CK and CD68 immunostains were negative in the right breast tumor (Fig. 3A-C).

\section{Discussion}

Malignant granular cell tumors are similar in epidemiology to their benign counterparts. Both are found twice as often in females as in males and occur most commonly between the age of 40 and 69 (2). Initially, granular cell tumors were considered to arise from histiocytes, fibroblasts, myocytes or intestinal mesenchymal cells. On the basis of ultrastructural observations and histochemical evidence, the tumors are now widely accepted to originate from Schwann cells (7). Larger tumor size, advanced age and local recurrence at presentation correlate with a worse prognosis (8). MGCT is an extremely rare type of cancer and at present no more than 100 cases have been described in the English language literature. Among them, many cases lack adequate follow-up. Consequently, diagnostic criteria and management strategy for MGCTs remain controversial (9).

It is difficult to distinguish between malignant and benign GCTs. Although metastasis remains one of the most important criteria for defining malignancy, there is general recognition that not all malignant tumors, even those of high grade, actually metastasize. Clinically, it is of great significance to define malignancy before metastasis occurs. Six histological criteria have been established to predict malignant behavior according to the retrospective analysis of previous case reports of malignant GCTs. These are spindling of the tumor cells, the presence of vesicular nuclei with large nucleoli, increased mitotic rate ( 2 mitoses per 10 high-power fields at $x 200$ magnification), a high nuclear to cytoplasmic ratio, pleomorphism and necrosis (10). Neoplasms that meet three or more of these criteria are classified as malignant, those that meet one or two criteria are classified as atypical, and those that exhibit only focal pleomorphism are classified as benign (11). In the reported case study, the two initial lesions were consistent with benign histological performance according to the stated criteria. The later lesions were classified as malignant due to the observation of spindling of the tumor cells, vesicular nuclei with large nucleoli and increased mitotic rate. The benign lesion existed for several years and recurred twice following local resection. Ultimately, multiple subcutaneous and lymph node metastases occurred. Therefore, we presume that MGCT results from the malignant transformation of benign GCT. Although the possibility that there were multiple primary GCTs (multifocality) cannot be excluded, metastasis is a logical explanation for the simultaneous appearance of MGCT in the right breast and 
right groin. In contrast to the common sites for distant metastases, which include bone, peripheral nerves, the peritoneal cavity and the lung (5), the present case involved metastases in the breast. To the best of our knowledge, this is the first case of MGCT with breast metastasis that has adequate follow-up. The right breast GCT closely resembled breast carcinoma in the ultrasound and X-ray examination; however, microscopic examination revealed significant differences between them. Since S100 is positive in one third of breast carcinomas and cytokeratin is only positive in breast carcinomas, cytokeratin immunohistochemical staining was performed to differentiate GCT from common malignant breast tumors.

Although there is considerable overlap in the $\mathrm{Ki}-67$ proliferative index between histologically benign, atypical and malignant GCTs $(11,12)$, statistical analysis reveals a correlation between the $\mathrm{Ki}-67$ proliferative index and malignant classification. A score of $>10 \%$ for the Ki-67 index was significantly correlated with malignancy and unfavorable prognosis. In the reported case, the previous two tumors of the abdominal wall had a Ki-67 proliferative index of $<1 \%$, which increased to $10 \%$ in the second recurrence and was associated with increased nuclear pleomorphism, vesicular nuclei with prominent nucleoli and increased mitotic rate. Similarly, Le et al reported that a clinical recurrence exhibited progressively more marked nuclear pleomorphism and vesicular nuclei with prominent nucleoli, as well as an increased Ki-67 proliferative index (1-10\%). Due to the absence of demonstrable metastases, the tumor was classified as atypical GCT of unknown malignancy (12). We believe that in GCT clinical recurrences, an increased Ki-67 proliferative index predicts clinical behavior and should be one of the criteria for defining malignancy.

Wide local excision with regional lymph node dissection is the first choice of treatment for MGCT. In metastatic patients, there is no evidence that resection of the metastatic lesions improves prognosis. In this case, considering the axillary lymph node metastases of the right breast metastatic lesion, regional lymph node dissection of the metastatic lesion was advised. Whether regional lymph node dissection improves prognosis may be determined by the follow-up. Although certain cases of successful treatment have been reported, the effectiveness of chemotherapy and radiotherapy remains controversial.

\section{References}

1. Abrikossoff A: Über Myome, ausgehend von der quergestreiften willkürlichen Muskulatur. Virchows Arch Pathol Anat Physiol 260: 215-233, 1926.

2. Mukai M: Immunohistochemical localization of S-100 protein and peripheral nerve myelin proteins ( $\mathrm{P} 2$ protein, $\mathrm{P} 0$ protein) in granular cell tumors. Am J Pathol 112: 139-146, 1983.

3. Gokaslan ST, Terzakis JA and Santagada EA: Malignant granular cell tumor. J Cutan Pathol 21: 363-370, 1994.

4. Lack EE, Worsham GF, Callihan MD, Crawford BE, Klappenbach S, Rowden G and Chun B: Granular cell tumor: a clinicopathological study of 110 patients. J Surg Oncol 13: 301-316, 1980.

5. Curtis BV, Calcaterra TC and Coulson WF: Multiple granular cell tumor: a case report and review of the literature. Head Neck 19: 634-637, 1997.

6. Uzoaru I, Filler B, Ray V, Hubbatd-Shepaid M and Rhee H: Malignant granular cell tumor. Arch Pathol Lab Med 116: 206, 1992.

7. Fisher ER and Wechsler H: Granular cell myoblastoma a misnomer. Electron microscopic and histochemical evidence concerning its Schwann cell derivation and nature (granular cell schwannoma). Cancer 15: 936-954, 1962.

8. Enzinger RM and Weiss SW (eds): Granular cell tumor. In: Soft Tissue Tumors. 4th edition. Mosby, St. Louis, pp1178-1206, 2001.

9. Mahoney A, Garg A, Wolpowitz D and Mahalingam M: Atypical granular cell tumor - apropos of a case with indeterminate malignant potential. Am J Dermatopathol 32: 370-373, 2010.

10. Khansur T, Balducci L and Tavassoli M: Granular cell tumor. Clinical spectrum of the benign and malignant entity. Cancer 60: 220-222, 1987.

11. Fanburg Smith JC, Meis Kindblom JM, Fante R and Kindblom LG: Malignant granular cell tumor of soft tissue: Diagnostic criteria and clinicopathologic correlation. Am J Surg Pathol 22: 779-794, 1998.

12. Le BH, Boyer PJ, Lewis JE and Kapadia SB: Granular cell tumor immunohistochemical assessment of inhibin-alpha, protein gene product 9.5, S100 protein, CD68, and Ki-67 proliferative index with clinical correlation. Arch Pathol Lab Med 128: 771-775, 2004. 